\title{
T-matrix many-particle theory for coherently coupled superlattice optics
}

\author{
T. Schmielau ${ }^{\mathrm{a}}$, M.F. Pereira Jr $\mathrm{Jr}^{\mathrm{b}}$, and K. Henneberger ${ }^{\mathrm{a}}$ \\ ${ }^{a}$ Fachbereich Physik, Universität Rostock, D-18051 Rostock, Germany \\ ${ }^{b}$ NMRC, University College Cork, prospect Row, Lee Maltings, Cork, Ireland
}

\begin{abstract}
The high-order Coulomb correlations described by T-matrix diagrams in both carrier occupation and polarization functions are studied here with the Keldysh-Green's Functions formalism. Numerical applications for low dimensional semiconductor systems illustrate the relevance of the effects and their importance for realistic nonlinear optical spectra calculations. A frequency and momentum resolved numerical demonstration of the Mott transition at the T-matrix level is presented.
\end{abstract}

Keywords: semiconductors, superlattices, T-matrix, many body effects, Green's functions.

Paper received 12.02.04; accepted for publication 17.06.04.

\section{Introduction}

Coulomb effects are now well established as the origin of the near band-gap optical nonlinearities in semiconductors [1], and many-body techniques are required for a realistic description of their optical and transport properties, due to the large number of charged carriers created. Among the current possibilities, non-equilibrium Keldysh-Green's functions allow the consistent description of both equilibrium and non equilibrium situations [2].

As the temperature is reduced, and the effective Coulomb interaction between charged carriers increases, especially in systems designed to enhance those effects, like low dimensional semiconductors, many-particle corrections of increasingly higher order are required to explain the electronic and highly non-linear optical properties of such systems, e.g. corrections beyond the Random Phase (RPA) and GW approximations must be considered [3,4,5,6].

Numerical results for deviations beyond RPA and GW have been given for the first time in Ref. 3, where excitoninduced satellites in the one-particle spectral functions of charged carriers in a bulk material are discussed.

In low-dimensional systems, quantum-confinement and bandstructure effects add a further complication for realistic numerics, and here we use as a model system, coherently coupled semiconductor superlattices, in which such complications are dealt with by means of an effective dimensionality formalism that describes the evolution of the system from quasi- two to quasi- three dimensions $[7,8]$.
We discuss under which conditions the $T$-matrix leads to deviations in the selfenergies that characterize the relevant quasi-particles of the system, and show a frequency and momentum resolve numerical demonstration of the Mott transition at the T-matrix level.

The paper is organized as follows: In Section 2 the main non-equilibrium Green's functions expressions are summarized. In Section 3 the dephasing computation is outlined. Numerical results are presented in Section 4, which is closed by a brief Summary.

\section{Nonequilibrium Green's functions expressions}

The interacting quasi-particles, which describe the excited semiconductor in our Keldysh-Green's, function approach, namely, carriers $(G)$, photons $(D)$, and plasmons $(W)$ [9], have their time-dependence dictated by Dyson equations (sum over repeated indices is assumed),

$$
\begin{aligned}
& {\left[G_{0, a c}^{-1}(13)-\Sigma_{a c}(13)\right] G_{c b}(32)=\delta_{a b}(12),} \\
& {\left[D_{0}^{-1}(13)-P(13)\right] D(32)=\delta_{T}(12),} \\
& {\left[W_{0}^{-1}(13)-p(13)\right] W(32)=\delta(12),}
\end{aligned}
$$

where $\delta_{T}$ is the transverse delta function. The labels $a, b, c \ldots$ denote generically denote generically the several conduction and valence sub bands. The inverse free propagators read 


$$
\begin{aligned}
& G_{0, a b}^{-1}(12)=\left[i \hbar \frac{\partial}{\partial t_{1}}-h_{e f f}(1)\right] \delta(12), \\
& W_{0}^{-1}(12)=-\frac{\varepsilon_{0}}{4 \pi e^{2}} \Delta_{1} \delta(12), \\
& D_{0}^{-1}(12)=\left[\Delta_{1}-\frac{1}{c^{2}} \frac{\partial^{2}}{\partial t_{1}^{2}}\right] \delta(12) .
\end{aligned}
$$

Here $\varepsilon_{0}$ is the static dielectric function. The effective one-particle Hamiltonian in the equation for the free-carrier propagator reads,

$$
h_{\text {eff }}(1)=\left[H_{0}(1)+\Phi_{\text {eff }}(1)\right] \delta_{a b}+\frac{i e \hbar}{c m_{0}} \vec{A}_{e f f}(1) \cdot \vec{\nabla}(1),
$$

where $\Phi_{\text {eff }}$ and $\vec{A}_{\text {eff }}$ denote, respectively, the expectation values of the scalar and vector potentials. The selfenergies, $\Sigma, P$ and $p$ are called, respectively the carrier selfenergy, the transverse and longitudinal polarization functions. Detailed band-structure and quantum-confinement effects are included in the theory through $h_{\text {eff }}$ in the free-carrier propagator, and also in the optical transition selection rules described by the matrix elements of the velocity operator,

$$
\vec{\Pi}(12)=\left(\vec{\Pi}(1)+\vec{\Pi}^{*}(2)\right) / 2=\hbar(\vec{\nabla}(1)-\vec{\nabla}(2)) / 2 i m_{0}
$$

The carrier self-energy $\Sigma$ leads to bandgap renormalization, includes dynamic effects such as corrections beyond Hartree-Fock, scattering rates in the carriers kinetics, and enables the description of bound states (excitons) in the spectral density of carriers, defining the degree of ionization. The longitudinal polarization function $p$ is responsible for (plasmon) screening of the Coulomb interaction. Furthermore, it describes dynamical screening, screening by excitons, plasmon kinetics and the build up of screening, although, these issues will not be addressed in this paper. The transverse polarization function $P$ yields the excitation dependent absorption coefficient and refractive index, and defines scattering rates (generation/recombination, respectively absorption/emission) in the photon kinetics. It is responsible for the inclusion of bound states (excitons) in the photons spectral density.

Functional derivative or diagrammatic techniques allow the consideration of increasingly higher order Coulomb corrections in the self-energies. In this paper, we consider T-matrix correlation contributions (c) beyond the Random Phase (RPA) and $G W$ approximations. For the carrier and photon self-energies, we obtain $[4,5]$,

$$
\begin{aligned}
& \Sigma_{a a}(12)=\Sigma_{a a}^{G W}(12)+\Sigma_{a a}^{c}(12), \\
& \Sigma_{a a}^{G W}(12)=-i \hbar G(12) W(12), \\
& \Sigma_{a a}^{c}(12)=-i T_{a b}^{\prime \prime}(1324) G(43), \\
& P_{e h}(12)=P_{0, e h}(12)- \\
& -\hbar G_{e e}(13) G_{h h}(14) T_{e h}(3456) G_{e e}(52) G_{h h}(62),
\end{aligned}
$$

where we have introduced the electron-hole quantity,

$$
P_{e h}(1234)=-i \hbar G_{e e}(13) G_{h h}(24),
$$

Within screened ladder approximation, the $T$-matrix,

$P_{e h}(1234)=P_{0, e h}(1234)+$

$+\frac{1}{\hbar} P_{0, e h}(1256) T_{0, e h}(5678) P_{e h}(7834)$,

satisfies the equation,

$T_{e h}(1234)=W_{e h}(12) \delta(13) \delta(24)-$

$-i W_{e h}(12) G_{e e}(15) G_{h h}(26) T_{e h}(5634)$.

The transverse and longitudinal polarization functions are connected through

$P_{a b}(12)=\frac{4 \pi e^{2}}{c^{2}} \vec{\pi}(2) \vec{\pi}(1) p(11 \pm 2)$.

\section{Dephasing}

The possibility of computing the dephasing and the corresponding bandgap shift that follows by Kramers-Kronig allows for further predictability in the theory, in contrast with a previous approach where formulas that simulate the fully computed dephasing have been used [10]. They appear directly in the spectral function $\bar{G}$, entering all equations presented above. After suitable Fourier transformations,

$\bar{G}(k, \omega)=\frac{\hbar \Gamma_{a}}{\left(\hbar \omega-E_{a}\right)^{2}+\hbar^{2} \Gamma_{a}^{2}}$,

where $E_{a}=\hbar \varepsilon_{a}-\mathfrak{R}\left\{\sum_{a a}^{r}(k, \omega)\right\}$, and $\hbar \Gamma_{a}=-\mathfrak{I}\left\{\sum_{a a}^{r}(k, \omega)\right\}$ denote, respectively, the renormalized energies and the dephasing rate. The correlation functions are given by

$G_{a a}^{<}(k, \omega)=\bar{G}_{a a}(k, \omega) f_{a}^{<}(\omega)$,

and $f_{a}^{<}(\omega)$ is a Fermi function. Algebraic manipulations lead to the GW dephasing,

$\Gamma_{a}(\vec{k}, \omega)=-\sum_{\vec{q}} \int_{-\infty}^{+\infty} \frac{d \Omega}{2 \pi}\left[n_{B}(\Omega)+f_{a}(\omega+\Omega)\right] \times$

$\times \bar{G}(\vec{k}+\vec{q}) \mathfrak{I}\left[W_{a a}(\vec{q}, \Omega)\right]$,

where $n_{B}(\Omega)$ is the Bose distribution function. Using the Kramers-Kroning relation, the real part of the retarded selfenergy is given by

$\mathfrak{R}\left\{\sum_{a a}^{r}(\vec{k}, \omega)\right\}=P \int_{-\infty}^{+\infty} \frac{d \Omega}{2 \pi} \frac{\Gamma_{n}(\vec{k}, \Omega)}{\Omega-\omega}$.

Here $P \int$ denotes a Cauchy principal value integral. Using the Optical theorem for the retarded potential,

$\mathfrak{I}\left\{W_{a a}(\vec{k}, \omega)\right\}=\sum_{b=e, h}\left|W_{a a}(\vec{k}, \omega)\right|^{2} \mathfrak{I}\left\{p_{b b}^{r}(\vec{k}, \omega)\right\}$,

together with the retarded longitudinal polarization function, 


\section{T. Schmielau et al.: T-matrix many-particle theory for coherently coupled superlattice ...}

$p_{b b}^{r}(\vec{k}, \omega)=-\sum_{\vec{q}} \int_{-\infty}^{+\infty} \frac{d \omega_{1}}{2 \pi} \frac{d \omega_{2}}{2 \pi} \bar{G}_{b b}\left(\vec{q}, \omega_{1}\right) \bar{G}_{b b}\left(\vec{q}+\vec{k}, \omega_{2}\right) \times$

$\times \frac{f_{b}^{<}\left(\omega_{1}\right)-f_{b}^{<}\left(\omega_{2}\right)}{\omega-\omega_{1}+\omega_{2}+i \delta}$

the self energies can be computed iteratively together with the corresponding quasi-chemical potentials, which characterize the distribution functions. In order to understand the full process and make a connection to less advanced iteration schemes, note that the average number of particles in a system of particles of type $a$ (electrons or holes) reads

$N_{a}=\int\left\langle\Psi_{a}^{+}(R) \Psi_{a}(R)\right\rangle d R$,

which can be written as

$N_{a}=2 \sum_{\vec{k}} \frac{d(\hbar \omega)}{\pi} \frac{\hbar \Gamma_{a}}{\left(\hbar \omega-E_{a}(k)\right)^{2}+\hbar^{2} \Gamma_{a}^{2}} \cdot f_{a}^{<}(\hbar \omega)$.

In the quasi-particle approximation $\hbar \Gamma_{a} \rightarrow 0$, we obtain the usual $N_{a}=2 \sum_{\bar{k}} f_{a}\left(E_{a}(k)\right)$.

At this point we include higher-order T-matrix corrections, following the prescription presented in the previous section. We must then include the terms below in the iterative scheme. The Fourier-transformed ladder or correlation T-matrix self energy has Keldysh components given by

$\Sigma_{a a}^{c,<}\left(\vec{p}_{1}, \omega\right)=$

$=2 i \sum_{\vec{p}_{2}} \frac{d \Omega}{2 \pi} T_{a b}^{\prime \prime}\left(\vec{p}, \vec{p}^{\prime}, \vec{P}, \omega+\Omega\right) \cdot G_{b}^{<}\left(\vec{p}_{2}, \Omega\right)$,

with analogous expressions for the ">" Keldysh components. For cases in which effective masses can be defined, as in the superalattice data used for the numerics used here, $\vec{p}=\frac{m_{b}}{m_{a}+m_{b}} \vec{p}_{1}-\frac{m_{a}}{m_{a}+m_{b}} \vec{p}_{2}$, and $\vec{P}=\vec{p}_{1}+\vec{p}_{2}$ In the statically screened ladder approximation, the Fourier-transformed T-matrix, $T_{a b}^{r}\left(\vec{p}, \vec{p}^{\prime}, \vec{P}, \omega\right)$, satisfies the Bether-Salpeter equation,

$$
\begin{aligned}
& T_{a b}^{r}\left(\vec{p}, \vec{p}^{\prime}, \vec{P}, \omega\right)=W_{a b}\left(\vec{p}-\vec{p}^{\prime}\right)+ \\
& +\sum_{\vec{p}^{\prime \prime}} W_{a b}\left(\vec{p}-\vec{p}^{\prime \prime}\right) \cdot g_{a b}^{r}\left(\vec{p}^{\prime \prime}, \vec{P}, \omega\right) \cdot T_{a b}^{r}\left(\vec{p}^{\prime \prime}, \vec{p}^{\prime}, \vec{P}, \omega\right),
\end{aligned}
$$

where the uncorrelated two-particle Green's function reads, $g^{<}(\vec{p}, \vec{P}, \omega)=i \int \frac{d \Omega}{2 \pi} G_{a}^{<}\left(\vec{p}_{1}, \omega-\Omega\right) \cdot G_{b}^{>}\left(\vec{p}_{2}, \Omega\right)$.

\section{Numerical results and discussion}

In this section, we apply our theory for coherently coupled superlattices, treated within the anisotropic medium ap-

SQO, 7(2), 2004

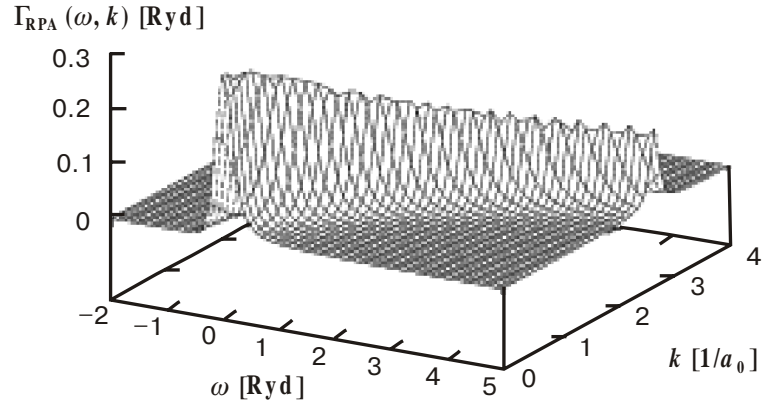

Fig. 1. GW dephasing for a $5 \AA \mathrm{ZnSe} / 75 \AA \mathrm{ZnMgSe}$ superlattice at $T=77 \mathrm{~K}$ and $N=10^{15}$ carriers $/ \mathrm{cm}^{3}$.

proach, in which the motion of electrons and holes is characterized by angle-averaged effective masses. Structures designed to increase the carriers confinement lead to larger effective masses [7,8].

Figure 1 displays the GW dephasing of electrons obtained from the imaginary part of the corresponding retarded selfenergy for a II-VI superlattice. Higher order corrections are due to T-matrix corrections and are depicted in Fig. 2. The GW dephasing follows the electronic dispersion (free carrier energy plus Fock and GW self energies), while the T-matrix dephasing is mainly centered about the excitonic dispersion. The correlations described by the T-matrix also lower the potential energy of the unbound carriers, thus increasing the damping on the low-energy side of the free dispersion and decreasing it on the highenergy side. This effectively shifts the spectral weight to lower energies. In all plots the energy axis is the detuning with respect to the fundamental band gap (including the quantum confinement correction) and measured in units of the superlattice binding energy.

Figure 3 illustrates the bleaching of the excitonic absorption (Mott transition) at the T-matrix level for

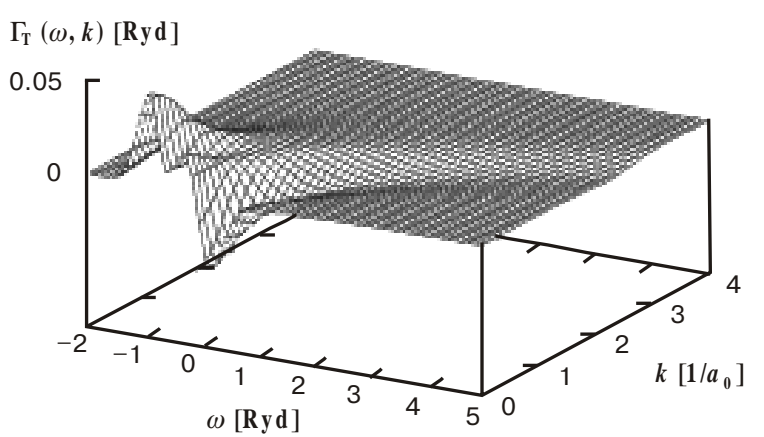

Fig. 2. T-matrix dephasing for the superlattice in Fig. 1, evaluated with the same parameters. 

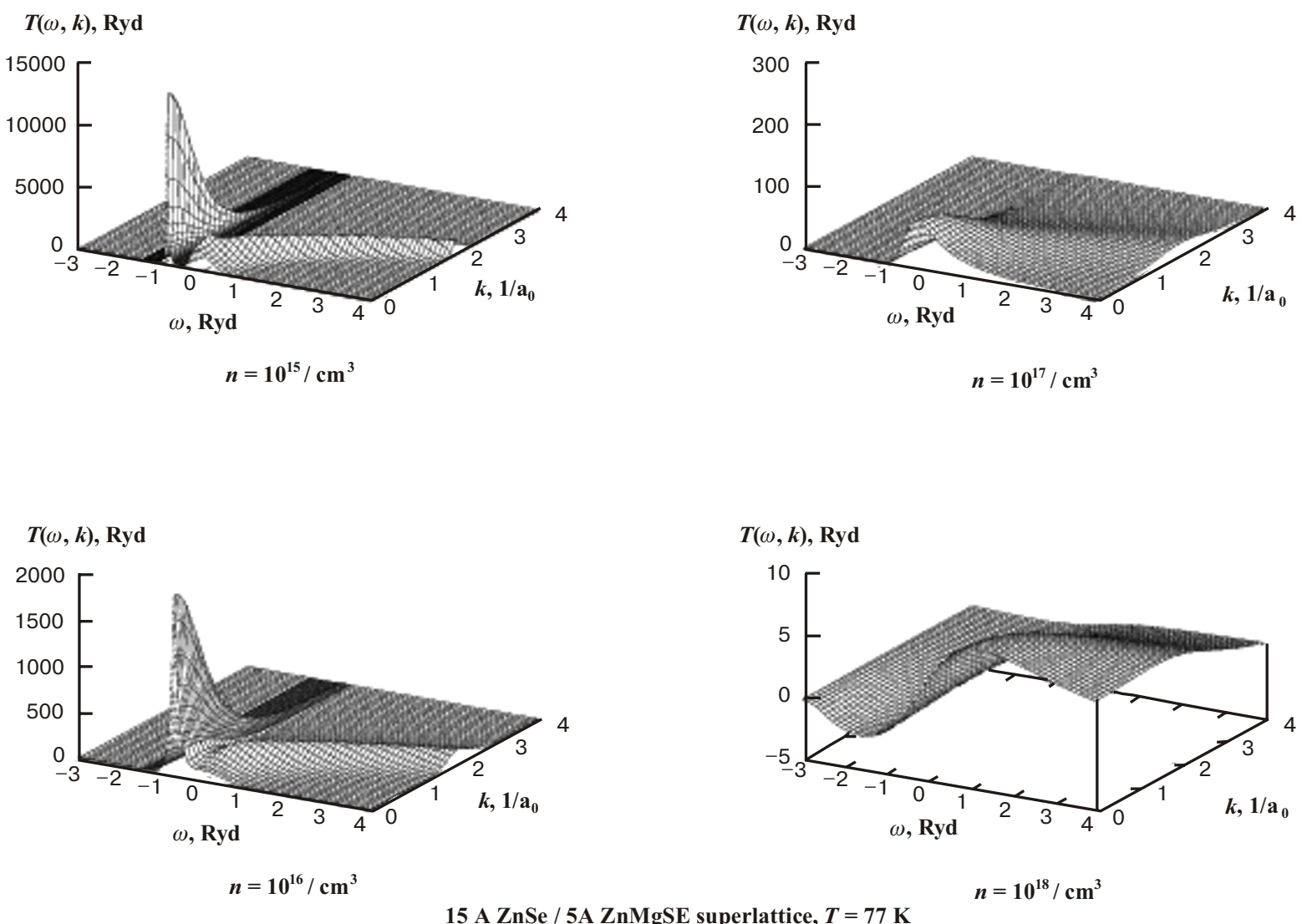

Fig. 3. Evolution with increasing density of the T-matrix for a $5 \AA$ $/ 15 \AA \mathrm{ZnSe}-\mathrm{ZnMgSe}$ superlattice.

$15 \AA$ / $5 \AA$ GaAs superlattice at $150 \mathrm{~K}$. The lowest density plot clearly displays contributions from the 1 s bound excitonic state, as well as the combined contribution of the $2 s$ and $2 p$ states, which already overlaps with the continuum. The $k$-dependence of these features reflects the corresponding hydrogen wavefunctions, thus the $2 s$ and $2 p$ features have a maximum at $k>0$. While the $2 p$ contribution is suppressed in the optical properties like the polarization function, it does appear in the T-matrix in forward scattering, which enters the T-matrix selfenergy. It occurs in our numerical calculations only because no further angle averaging is performed upon the $\mathrm{T}$-matrix elements. As the carrier density increases from $N=10^{15}$ to $10^{18}$ carriers $/ \mathrm{cm}^{3}$, the band-gap shrinkage shifts the continuum towards lower energies, where it joins the excitonic features (Mott transition). Our calculations are capable of displaying a frequency- and momentum resolved numerical demonstration of the Mott transition.

The sign change in the T-matrix at $10^{18}$ carriers $/ \mathrm{cm}^{3}$ corresponds to the appearance of gain in the optical spectra.

Figure 4 shows distribution functions for a $5 \AA / 15 \AA$ $\mathrm{ZnSe}-\mathrm{ZnMgSe}$ superlattice.

The top, central and lower plots are, respectively for $T=77,150$ and $300 \mathrm{~K}$. On the left and right, $N=10^{15}$ and $10^{16}$ carriers $/ \mathrm{cm}^{3}$. The solid curves and circles are, respectively, for Fermi and Wigner distributions. For comparison, exciton distributions are also shown as dot- dashed curves. They are defined as the convolution of the squared $1 s$ wavefunction with the center-of-mass Boltzmann distribution.

Note that, as the temperature increases, the Fermi and Wigner distributions become indistinguishable. In other words, in this case, we can compute optical spectra without the T-matrix diagram in the carrier's self-energy, which justifies successful calculations by the present authors and several others in the literature for high temperatures considering Fermi distributions only.

Figure 5 displays the optical absorption with the T-matrix included on both polarization and self-energy diagrams. No phenomenological parameters are needed here in order to give a consistent broadening to the low-density spectra. Even if fully computed within the GW approximation, the broadening would be unrealistically small at low densities and an arbitrary dephasing would have to be added. In other words, our calculations add further predictability to previous approaches, extending rather successful approaches, which at high densities could reproduce several important experimental findings [11], to the low-density regime dominated by excitonic features.

In summary, the microscopic theory for the nonlinear optical properties of semiconductors presented here provides a technique to study Coulomb effects beyond RPA and GW, by analyzing their influence on optical spectra. Unphysical features, like a spurious absorption for 
T. Schmielau et al.: T-matrix many-particle theory for coherently coupled superlattice ...
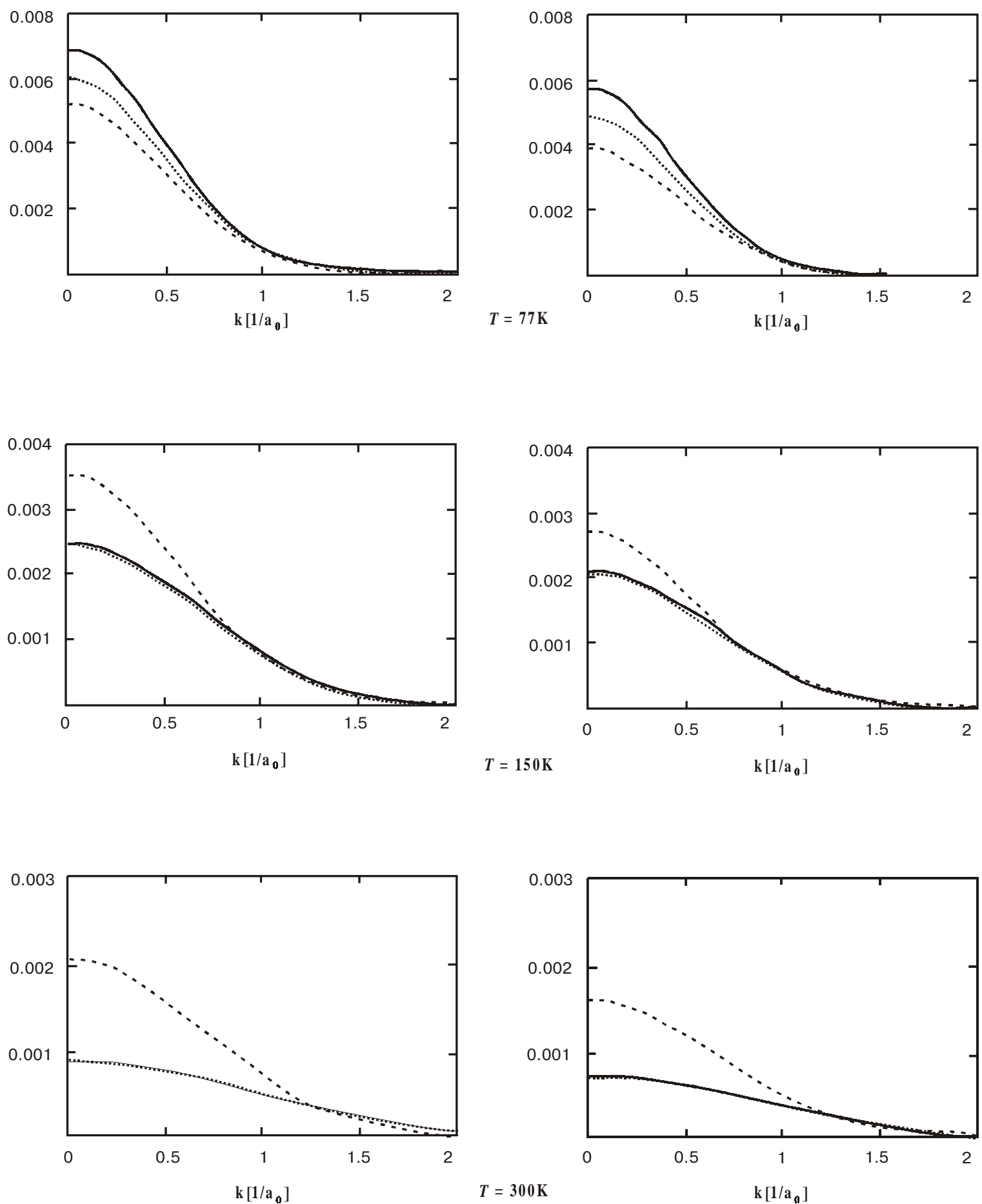

Fig. 4. Distributions functions for a $5 \AA$ / $15 \AA$ (left) and $5 \AA / 75 \AA \mathrm{ZnSe}-\mathrm{ZnMgSe}$ superlattices. The top, central and lower plots are, respectively for $T=77,150$ and $300 \mathrm{~K}$. The carrier density is $N=10^{15}$, and $10^{16}$ carriers $/ \mathrm{cm}^{3}$. Solid: Fermi distributions; Dot-dashed: Exciton distributions; Circles: Wigner distributions. 
T. Schmielau et al.: T-matrix many-particle theory for coherently coupled superlattice ...

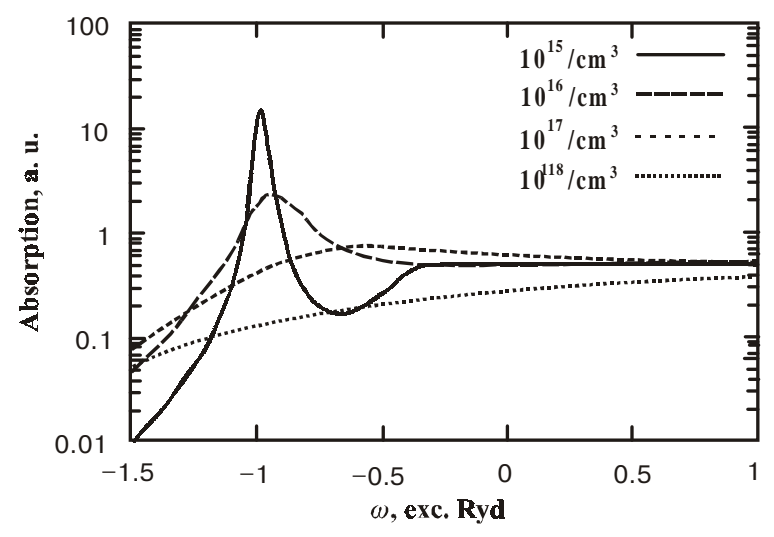

Fig. 5. Optical absorption for a $5 \AA / 15 \AA \mathrm{ZnSe}-\mathrm{ZnMgSe}$ superlattice at $T=300 \mathrm{~K}$. The solid, long-dashed, short-dashed and dotted curves are, respectively, for $N=10^{15}, 10^{16}, 10^{17}$, and $10^{18}$ carriers $/ \mathrm{cm}^{3}$.

photon frequencies below those in the gain range do not appear, since our polarization function satisfies the KMS sum rule. The numerical results show that the actual carrier occupation functions (Wigner distributions) differ from the commonly used Fermi distributions for sufficiently low carrier densities and temperatures. Our iterated GW and T-matrix dephasing adds further predictability to the approach and for multi-sub-band quantum wells provides important insight on high-density gain operation, which may be important for high-power semiconductor laser applications.
The numerics presented can also be used as the starting point for the realistic simulation of more complicated light emitting and processing devices.

\section{Acknowledgements}

The authors thank Science Foundation Ireland (SFI) and the Deutsche Forschungsgemeinschaft (DFG) for financial support of this work.

\section{References}

1. H. Haug, S.W. Koch, Quantum Theory of the Optical and Electronic Properties of Semiconductors, World Scientific, Singapore (1990).

2. Progress in Nonequilibrium Green's Functions, Edited by M. Bonitz, World Scientific, Singapore, (2000).

3. R. Zimmermann, Many-Particle Theory of Highly Excited Semiconductors, "Teubner Texte zur Physik", Band 18, Leipzig (1987), ISBN 3-322-00493-7.

4. R. Schepe, T. Schmielau, D. Tamme, and K. Henneberger // Phys. Stat. Sol. (b), 206, pp. 273 (1998).

5. M. Pereira Jr. and K. Henneberger // Phys. Rev. B, 58 pp. 2064 (1998).

6. T. Schmielau, G. Manzke, D. Tamme, and K. Henneberger, Phys. Stat. Sol. (b), 221, pp. 215 (2000).

7. M. Pereira Jr., I. Galbraith, S. Koch, and G. Duggan // Phys. Rev. B, 42, pp. 7084 (1990)

8. M. Pereira Jr. // Phys. Rev. B, 52, pp. 1978 (1995).

9. K. Henneberger and H. Haug // Phys. Rev. B, 38, (1988).

10. M.F. Pereira Jr., R. Binder and S.W. Koch // Appl. Phys. Lett., 64, pp. 279 (1994).

11. P. Michler, M. Vehse, J. Gutowski, M. Behringer, and D. Hommel, M.F.Pereira Jr. and K. Henneberger // Phys. Rev. B, 58, pp. 2055 (1998). 